lich fie Buttermilch, die disperse Phase." Diese Ansicht ist falsch, denn die sichtbaren Hollen zeigen, das das Fett keine kontinuierliche Phase ist.

Diese Membranen in der Butter entsprechen ganx den zarten festen Häutchen, welche Ramsden (loc. cit.) bei der Emulsion von Oliwenöl in Saponinlösung bemerkte. Sie besteben aus dem infolge der niedrigen Oberflächenspannung sich an den Grenzflächen verdichtenden Kolloid und sind nur urter bestimmten optischen Bedingungen sichibar. Ihre
Exisienz ist ebenfalls durch die Soxhlet'sche Theorie nichr erklärbar.

Die Entstehung von Milchschaum und Schlagsalne, die Bildung einer festen Haut beim Erbitzen der Milch, die Entsiehung von Butter beim Bearbeiten des Rahns lassen sich alle durch die Annahme eines Schaumstoffes in der Miich, der vermutlich ein Eiweihstoff ist, erklären. Der Beweis für die Existenz dieses Stoffes in der Milch darf als gegeben betrachtet werden, wennschon seine Reindarstellung noch nicht geglückt ist.

\title{
Ueber die elektrolytische Alkalientziehung aus Salzen.
}

Von A. Lottermoser (Dresden.)

(Eingagangen am 4. Mărz 1922.)
Die kurze Veröffentlichung von M. Krög e : ) über die Darstellung von Kieselsäure- und Wolframhydroxydsolen mit Hilfe der Hildebrandzelle veranlabt mich, hinzuweisen auf Untersuchungen, welche im Laboratorium vo $\mathrm{Kn}$ or re's in den Jahren 1899, 1903 und 1905 ausgeführt worden sind und auf meine Versuche, die ich 1920 begonnen hatte, ohne die früheren Arbeiten zu kennen. Da ich beabsichtige über den Gegenstand weiter zu arbeiten, möchte ich etwas näher auf das bisher Erreichte eingehen.

Es ist bekannt, dab man durch Elektrolyse einer Chromatiösung mit Diaphragma zu Bichromat gelangt, da dem Chromat Akali entzogen wird. Dieser Vorgang ist besonders eingehend von Erich Mulle ${ }^{2}$ ) studiert worden. Ich habe vor kurzem mit Dipl.-Ing. Kurt Falk über diesen Prozeß weitere Versuche angestellt, um besonders, d̈e Bedingungen für die besten kathodischen und anodischen Stromausbeuten und ihren zeitlichen Verlauf zu studieren. Ueber die Einzelheiten dieser Untersuchungen wird an anderer Stelle berichtet, hier soll nur so viel angedeutet werden, dab bei Anwendung von Tondiaphragmen die Ausbeuten an Anode und Kathode wesentlich von den Konzentrationen and Ueberführungszahlen der anweseriden Elektrolyte abhängig sind, die günstigsten Bedingungen bei Zwischenspaltung eines besonderen Mittelraumes zwischen Kathoden- und Anodenraum und Fällung sämtlicher Räume mit derselben Chromatlösung obwalten und der

1) M. Kröget, Koll.-Zeitschr. 30, 16 (1922).

3) Erich Muller u. Sauer, Zeitschr. f. Elektrochem. 18, 844.(1912); Erich Mïlier, Elektrochem. Praktikam, 3. Auff. (Dresden u. Leipzig), 179.
Prozeb seicht so weit fortgesetzt werden kann, bis in Anodenraume nur noch Chromsäure enthalten ist, cin Zustand, der sich durch rapiden Anstieg der Klemmenspannung bei konstant gehaltener Stromstärke kennzeichnet. Indessen ist die Anwendung von Diaphragmen durchaus nicht Bedingung für die Durchführung des Prozesses, sondern er verläurt in demselben Sinne, werin auch selbstverständlich mit sehr ungünstigen Anoden- und Kathodenausbeuten, wenn man eine Art Glockenapparat anwendet.

An Molybdater und Woltramaten sind Elektrolysen mit Diaphragma von EItzbacher, Terwelp, Schaefer und Junius ${ }^{3}$ im Knorre'schen Laboratorium ausgefinhrt worden. Sie haben dabei aus gewöhnlichen Molybdaten und Wolframaten Para- und Metamolybdate, bzw. -wolframate, ja selbst die freien Säuren gewonnen, doch haben sie keine Versuche über den Gang der Elektrolyse, vor allem keine Vorausberechnung dartiber angestellt, wieviel Alkali in dem Kathodenraum durch Entladung und Ueberführung entstanden sein mub, um im Anodenraum ein Salz bestimmter $\mathrm{Zu}$ sammensetzung zu erhalten. Vielmehr haben sie zur Darstellung der Parasalze die Elektrolyse bis nahe an eine neutrale Reaktion der Anodenflüssigkeit (Natrium- und Kaliumsaize der normalen Säuren reagieren bekanntlich in Lösung durch Hydrolyse alkalisch) zur Gewinnung der Metasalze bis zur sauren Reaktion der Lösung fortgeführt und endlich Abscheidung der Säuren bei sehr lange fortgesetzter Elektrolyse erreicht.

3) Dissertationen, Berlin 1899, 1908,1903 u. 1905; Schaefer, Zeitschr. f. anorg. Chem. 38, 174 (1904); Junias, Zeitschr. f. Anorg. Chem. 46, 428 (1905). 
Ich habe nun diese quantitativen Untersuchungen zunächst am normalen Wolframat $\mathrm{Na}_{2} \mathrm{WO}_{4} \cdot 2 \mathrm{H}_{2} \mathrm{O}$ in Angrift genommen und bin zuerst aut die Gewinnuris von Parawolframat ausgegangen. Die Versuchsanordnung war die folgende: Eine Tonzelle aus Berliner Porzellan von ungefâhr $250 \mathrm{ccm}$ Fassungsraum wurde mit einer Lösung von $51 \mathrm{~g} \mathrm{Na} \mathrm{WO}_{4} \cdot 2 \mathrm{H}_{2} \mathrm{O}$ mit $\mathrm{H}_{2} \mathrm{O}$ auf 200 ccr gelost beschickt; in diese Lösung tauchte die zu einen Zylinder gebogene Platinanode. Die Zelle stand in einem weiten Becher, velcher mit $750 \mathrm{ccm}$ einer genan $1 / 10$ norm. NaOH beschickt war. Zur Vermeidurg einer Elektronsmose wurde die Flissigkeit im Anodenrawn $2 \mathrm{ccm}$ tiefer als im Kathodenratme gestellt. Die Elektrolyse wurde mit 2 Aupère unter gleichzeitiger Einschaltung eines Kupterkoulometers durchgeführt.

Die Berechnung der elektrolytischen Umwandlung des normalen Wolframates in Parawolframat gestaltet sich unter den beschriebenen Verhaitnissen folgendermaben:

$12 \mathrm{Na}_{2} \mathrm{WO}_{4}+7 \mathrm{H}_{2} \mathrm{O}=14 \mathrm{NaOH}+\mathrm{Na}_{10} \mathrm{~W}_{12} \mathrm{O}_{41}$

d. h. 6 Molen normalen Wolframats müssen $7 \mathrm{Mol} \mathrm{NaOH}$ entzogen werden, damit jenes im Parawolframat von obiger Fomel ibbergeht. $\mathrm{Na}_{2} \mathrm{WO}_{4} \cdot 2 \mathrm{H}_{2} \mathrm{O}$ hat das Mol.-Gew. 330, somit künnen wir die Proportion aufstellen: 6.330 $: 70000=51: \mathrm{x}$, wenn wir das entzogene Al. kali in $\mathrm{ccm} 1 / 10$ norm. Lösung ausdrücken. $x$ ist dann $1803 \mathrm{ccm} 1 / 10$ rorm., die zu dem schon im Kathoden aum befindlichen $750 \mathrm{ccm} 1 / 10$ norm. hinzugekommen sein müssen, wenn der Uebergang des Wolframats in Parasalz beendet ist. Nimmt man nun, wie es im allgemeinen iiblich ist, an, da\& zehn Proz. der gebildeten Natronlauge in den Poren des Diaphragmas verbleiben, so tritt eine Menge $\mathrm{NaOH}=1623 \mathrm{~cm} 1 / 10$ norm. in den Kathodenraum iber. Der Versuch muB also abgebrochen werden, wenn die Gesamtalkalimenge im Kathodenraume 2373 ccm 1/10 norm. beträgt. $\mathrm{Da}$ nun zur Ueberwachung des Portganges der Reaktion 2 ccm der Kathodenlauge titriert werden, müssen diese bei SchluB des Versuches $6,33 \mathrm{ccm} 1 / 10$ norm. entsprechen.

Gibt man aber dem Parawolframat die Formel $\mathrm{Na}_{6} \mathrm{~W}_{7} \mathrm{O}_{24} \cdot 16 \mathrm{H}_{2} \mathrm{O}$, so gestaltet sich die anaioge Rechnung so: $7 \mathrm{Na}_{2} \mathrm{WO}_{4}+4 \mathrm{H}_{2} \mathrm{O}$ $=8 \mathrm{NaOH}+\mathrm{Na}_{6} \mathrm{~W}_{7} \mathrm{O}_{24}$. Aus $51 \mathrm{~g}$ Wolframat. mübte sich theoretisch $\mathrm{NaOH}=1766,2 \mathrm{ccm}$ $1 / 10$ norm. ergeben, das ist für $2 \mathrm{ccm}$ Kathodenlauge $6,24 \mathrm{ccm} 1 / 10$ norm.

Rund sollien also am Ende $2 \mathrm{~cm}$ Kathodenlauge $6,30 \mathrm{~cm} 1 / 10$ norm. entsprechen.
Nach beendetem Versuche kristallisierten aus der Anodenlösung große, glasglänzende Kristalle aus, die schon dem äuberen Aussehen nach als solche des Parawolframates erkannt wurden. Eine Kristallwasser- und Wolframbestimmung bestätigte dies. Die Wolframbestimmung geschah nach dem auch im Treadwell beschriebenen, ausgezeichneten und leicht ausfuhrbaren Benzidinverfahren.

$0,7970 \mathrm{~g}$ des luftrockenen Salzes gaben

$0,1363 \mathrm{~g} \mathrm{H}_{2} \mathrm{O}=13,63$ Proz.

$0,5590 \mathrm{~g}$ des lufttrockenen Saizes gaben $0,4342 \mathrm{~g} \mathrm{WO}=77,69$ Proz.

$0,4.769 \mathrm{~g}$ des lufttrockenen Salzes gaben $0,3704 \mathrm{~g} \mathrm{WO}=77,66$ Proz.

Es zeigte sich nun, daß auch dann das Parawolframat auskristallisierte, als daranf ausgegangen wurde, das Diwolframat aus dem normalen Wolframat darzustellen. Die Reartion ist die folgende: $2 \mathrm{Na}_{2} \mathrm{WO}_{4}+\mathrm{H}_{2} \mathrm{O}=2 \mathrm{NaOH}$ $+\mathrm{Na}_{2} \mathrm{~W}_{2} \mathrm{O}_{7}$, auf ein Mol Wolframat entsteht also ein Mol $\mathrm{NaOH}$.

Die Berechnung ergibt, dab unter ben gleichen Verhältnissen. wie oben angegeben, $2 \mathrm{ccm}$ Kathoderiatuge nach Beendigung des Prozesses $5,71 \mathrm{~cm} 1 / 10$ norm. entsprechen mithter. Bei der Ausfuhrung der Elektrolyse war dieselbe aber versehentich etwas zu weit, $a$. h. bis zu dem Punkte $2 \mathrm{~cm}$ Kathodenlauge $=5,96 \mathrm{~cm}$ $1 / 10$ norm. gegangen. Beim Stehen schied die Kathodenlauge Kristalle desselben Aussehens wie beim oben beschriebenen Versuche aus. The Analyse ergab:

$1,1041 \mathrm{~g}$ lufturockenen Salzes lieferten

$0,7967 \mathrm{~g}$ lufttrockenen Salzes gaben $0,1226 \mathrm{~g} \mathrm{H}_{2} \mathrm{O}=15,39$ Proz.

Die theoretischen Zahlen für das $\mathrm{Salz}$ $\mathrm{Na}_{10} \mathrm{~W}_{12} \mathrm{O}_{41} .28 \mathrm{H}_{2} \mathrm{O}$ sind für $\mathrm{WO}_{3}=77,37$ Proz., für $\mathrm{H}_{2} \mathrm{O}=14,02$ Proz., für das Salz $\mathrm{Na}_{6} \mathrm{~W}_{7} \mathrm{O}_{24}$ $.16 \mathrm{H}_{2} \mathrm{O}$ auf $\mathrm{WO}_{3}=77,40$ Proz., auf $\mathrm{H}_{2} \mathrm{O}$ $=13,73$ Proz. Dem bei dem letzten Versuche entstandenen Salze könnte vielleicht eine Spur Metawolframat beigemischt sein: $\mathrm{Na}_{2} \mathrm{~W}_{4} \mathrm{O}_{13}$ $.10 \mathrm{H}_{2} \mathrm{O}$ enthält theoretisch 79,30 Proz. $\mathrm{WO}_{3}$ und 15,4 Proz. $\mathrm{H}_{2} \mathrm{O}$.

Die Dimensionen des angewendeten Apparates sind nun angesichts des wertvollen $z u$ verarbeitenden Materiales reichiich groß, ich habe deshalb denselben bei weiteren Versuchen durch einen bedeutend kleineren ersetzt, dessen Anodenraum, also das Innere der zylindrischen Tonzelle, $35-40 \mathrm{ccm}$ aufnehmen konnte und dessen Kathodenraum $300 \mathrm{~cm} 1 / 10$ norm. Natron- 
lange faBte. Darin sollte zuerst Natriumtriwolframat $\mathrm{Na}_{4} \mathrm{~W}_{3} \mathrm{O}_{11} .7 \mathrm{H}_{2} \mathrm{O}$ dargestelit werden. Die Umsetzung wäre die folgende; $3 \mathrm{Na}_{2} \mathrm{Wo}_{3}$ $-\mathrm{H}_{2} \mathrm{O}=2 \mathrm{NaOH}+\mathrm{Na}_{4} \mathrm{~W}_{3} \mathrm{O}_{11}$ und $2 \mathrm{~cm}$ der Kathodenlauge sollten $=3,63 \mathrm{ccm} 1 / 10$ norm. sein. Die Titration ergab am Schlub $2 \mathrm{com}$ Kathodenlauge $=3,78 \mathrm{ccm} 1 / 10$ norm, aiso ein wenig zu viel. Es kristallisierte abermals Parawolframat aus der Anodenlösung aus:

$0,5139 \mathrm{~g}$ Subst gaben $0,3990 \mathrm{~g} \mathrm{WO}_{3}$ oder 77,64 Proz, obgleich zur völligen Unwandlung des normalen Woltramates in Parawolframat am Schlusse $2 \mathrm{ccm}=4,87 \mathrm{ccm} 1 / 10$ norm. hätten sein milssen, also rund $1 \mathrm{ccm} 1 / 10$ norm. mehr für je 2 ccra hätten entstehen soilen. Das kann nicht anders gedeutet werden, als daB Natriumparawollramat als Bodenkörper auch mit Lösungen im Gleichgewicht sich befindet, in denen das Verhältnis von $\mathrm{NaOH}: \mathrm{WO}_{3}$ ein gröberes ist als 5:6 oder 6:7 wie im Parawoliramat, z. B. wit Lösungen $\mathrm{NaOH}: \mathrm{WO}_{3}$ wie $1: 1$ oder gar 4:3. Meines Wissens sind bisher die Gleichgewichte zwischen $\mathrm{NaOH}, \mathrm{WO}_{3}$ und Bodenkörpern noch nicht systematisch untersucht worden, würden sich woh! auch nicht leicht untersuchen lassen; aber die hier angegebene Methode des Entzuges von Alkali durch Elektrolyse aus Wolframatlösungen bietet die Möglichkeit, beliebige Verhältnisse zwischen $\mathrm{NaOH}$ und $\mathrm{WO}_{3}$ in der Lösung herzustellen. Ich habe Herrn cand. chem. Fla hh zur Untersuchung dieser Verhältnisse veranlaBt.

Die Methode der elektrolytischen Ansäuerung ist einet sehr vielseitigen Anwendung fühig, sie iabt sich nicht nur auf Wolframate und Molybdate, sondern auch auf Vanadate, Tantalate, Niobate, ferner alle Salze, welche auch saure Salze bilden können, wie Sulfate, Phos- phate, Arseniate usf. anwenden und soll dazu verwendet werden.

Was endlich die Möglichkeit der Darstellung kolloider Sauren betrifft, so hat Schaefer ${ }^{4}$ ) in seiner Dissertation ausgefihirt, dab durch eine Tag und Nacht fortgesetzte. Elektrolyse mit Diaphragma die Anodenlösung in einen dicken Brei von gelber Wolframsäure verwandelt worden sei. Nun hat $S c h$ a ef e r eine gesättigte Lösung von Natriumwolframat zur Elektrolyse verwendet, und es ist die Möglichkeit nicht von der Hand $z$ weisen, daB man in wesentlich verdünnterer Lösung $z u$ einer kolloiden Lösung yon Wolfrainsäure würde ebenso gelangen können, wie es $\mathrm{Kröger}$ mit der Hildebrandtzelle geiungen ist, mit dem wesentlichen Unterschiede, das im Falle der Anwendung des Diaphragmas eine kathodische Reduktion nicht in Betracht kommt. Auch diese Versuche sollen an einer ganzen Reihe von Salzen, z. B. Silikaten, Wolframaten, Molybdaten usw. in Angriff genommen werden.

Damit ist abet die Fülle der Möglichkeiten noch nicht erschöpft; vielmehr läBt sich auch der Vorgang der Entziehung von Alkali umgekehrt denken, d. h. man könnte eine Entziehung von Săure auf elektrolytischem Wege herbeiführen. Dadurch würden basische Salze gebildet werden. Allerdings liebe sich dieser Prozeb nur dort verwirklichen, wo die Kathode keine Reduktionswirkungen herbeiführen kann, die störende Beimengungen erzeugen würden. An Aluminium-, Chromi-, Thorium-und Zirkonsalzen soll der Versuch zur Durchführung dieses Gedankens in Angriff genommen werden.

Dresden, Februar 1922.

Technische Hochsolule.

4) Sehater, loc cit.

\section{Referate.}

Arbeiten über allgemeine Kapillarchemie.

Bancrolt, W.D., Einige Probleme der Kontaktkatalyse. (Trans. Amer. Elektr. Soc. 36, 139, 1920.) Als Beispiel einer spezîíschen Wirkung: Trichlorathylchlorformiat wird durch $\mathrm{Al}_{2} \mathrm{O}_{3}$ in $\mathrm{CO}_{4}+\mathrm{CCl}_{4}$ zerlegt; durch $\mathrm{Fe}_{2} \mathrm{O}_{3}$ in Phosgen. Auch hier tritt ein solches Gleichgewicht ein, wenn das Reaktionsprodukt nicht ständig entfernt wird. R. E. Lg.

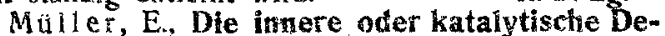
hydroxydation des Formaldehyds, (Zeitschr. f. Elektrochemie 27, 558, 1921.)

Bei Zugabe von fein verteiten Metallen wie Cu, $\mathrm{Ag}, \mathrm{Pd}$ oder Pt zu alkalischen Formaidehyd werden geringe Mengen $\mathrm{H}_{2}$ entwickeit. Stirmisch wird diese
Ho-Entwicklung bei Zugabe von kolloider Rhodiums lösung. $30 \mathrm{ccm} 15 \mathrm{n} \mathrm{NaOH}+50 \mathrm{ccm} 20$ prozentige$\mathrm{CH}_{2} \mathrm{O}$ geben mit $0,05 \mathrm{~g} \mathrm{Rh}$ bei 250 in zwei Stunden drei Liter $\mathrm{H}_{2}$. Daneben bildet sich Ameisensäure. Aennliche Dehydroxydationen sind bei einigen Alkoholen möglich (ibid. 563!.

R. E. Lg.

Reboul, G., u. R. Luce, Bedeutung der geometrischen Gestalt fester Körper auf die chemischen Veränderungen, welche'sie erleiden. (Compr. rend. $72,917,1921$.

Platten, Prismen. Pyramiden usw. von $\mathrm{Ag}$ oder Cu wurden in $\mathrm{H}_{2} \mathrm{~S}_{m}$, Sulfid-, $\mathrm{Cl}_{2}, \mathrm{Br}_{2}, \mathrm{~J}_{2}$-haltige Lösungen gebracht. Stets war dort die Reaktionsgeschwindigkeit (Dicke der umgewandelten Schicht, ge- 